\title{
An Energy Aware Heuristic-based Routing Protocol in Wireless Sensor Networks
}

\author{
Kamanashis Biswas ${ }^{1}$, Vallipuram Muthukkumarasamy ${ }^{1}$, Elankayer Sithirasenan ${ }^{1}$, Kalvinder Singh $^{2}$ \\ 1 School of ICT, Griffith University, Australia \\ Email: kamanashis.biswas@griffithuni.edu.au \\ \{v.muthu, e.sithirasenan\}@griffith.edu.au \\ 2 IBM, Australia Development Lab and Griffith University, Australia \\ Email: kalsingh@au.ibm.com
}

\begin{abstract}
Wireless Sensor Networks (WSNs) consist of small nodes equipped with sensing, data processing and radio transmission units. A number of clustering, routing, power management and data aggregation protocols are specially designed for WSNs where energy awareness is an essential design issue. Due to the resource constrained nature of sensor nodes, innovative techniques are required to extend the network lifetime in WSNs. This paper proposes an energy efficient routing protocol to find the forwarding path between source and destination node using heuristic function and $A^{*}$ search algorithm. Simulation results with OMNET++ show that our proposed protocol is efficient in terms of network lifetime, total energy dissipation and message throughput.
\end{abstract}

\section{INTRODUCTION}

Wireless Sensor Networks (WSNs) consist of hundreds or thousands of low-cost sensor nodes (SNs) which are randomly deployed in hostile environments [1], [2]. These nodes have finite on-board battery, small memory, limited computational power and communication bandwidth. Due to the resource constrained characteristics of $\mathrm{SNs}$, effective routing protocols have to be designed in order to maximize network lifetime and to ensure balanced energy consumption. Generally, in most of the routing protocols, the best path is selected for data transmission between source and destination node. But, if the same route is followed continuously then the forwarding nodes on that path will fail over a period of time [3], [4], [5].

In practice, many routing protocols minimize the total energy consumption by sacrificing uniform energy drainage in the networks [6]. These approaches cause network partition since most of the nodes following optimal routes quickly run out of energy. In worst case scenario, the network may become completely disfunctional as soon as the battery power of critical nodes is exhausted. Therefore, we propose an energy aware heuristic-based routing (EAHR) protocol to select the optimal route from source to destination node on the basis of a pre-defined threshold value on node energy. Our proposed EAHR protocol selects optimal route when the energy level of forwarding nodes is above the threshold level, otherwise it tries to find alternative routes to avoid the low energy nodes in order to extend network lifetime. The proposed routing protocol is suitable for home and office automation.

The rest of the paper is organized as follows. Section II describes the related works. Section III details the proposed heuristic-based routing protocol. Simulation and experimental results are discussed in Section IV. Finally, Section V concludes the paper.

\section{RELATED WORKS}

Karp et al. proposed a Greedy Perimeter Stateless Routing (GPSR) protocol that makes local packet forwarding decisions using a greedy algorithm [7]. The protocol makes greedy forwarding decisions using the router's immediate neighbours information in the network topology. However, in GPSR packets may be trapped in holes due to lack of adequate sensor density or various obstacles. To overcome the problem, the algorithm routes data packets around the perimeter of the region. However, the protocol still may be trapped in blind alley if the planner sub-graph used by the GPSR's perimeter mode is not connected.

Geographic and Energy Aware Routing (GEAR) protocol uses geographically informed neighbour selection heuristics to forward a packet towards the target region [8]. Each node keeps an estimated cost and a learning cost of reaching the destination through its neighbours. The estimated cost is a combination of residual energy and distance to the destination. The learned cost is a refinement of the estimated cost that accounts for routing around holes in the network. A hole occurs when a node does not have any closer neighbour to the target region than itself. The learned cost is propagated one hop back every time a packet reaches the destination so that route setup for next packet will be adjusted. GEAR performs better than GPSR in terms of throughput and energy consumption. But, the protocol is basically designed for ad-hoc networks and does not support scalability and mobility issues.

WSNHA-GAHR is a greedy and $\mathrm{A}^{*}$ heuristic routing algorithm for wireless sensor networks in home automation [9]. The algorithm uses greedy forwarding technique based on the smallest Euclidean distance to minimize the number of hops for data transmission. Furthermore, to overcome the local minimum problem and unpredictable changes in topology, the protocol automatically adjusts the route using the $\mathrm{A}^{*}$ search algorithm. The main drawback of GAHR protocol is that it does not consider residual energy of sensor nodes. Hence, if a node with low energy is selected for routing data packets, it may deplete its energy quick.

In [10] and [11], the authors presented two different approaches for extending network lifetime in WSNs. To ensure sustainability of sensor nodes, the protocols distribute the traffic load among the nodes having much residual energy. Both routing schemes achieve energy efficiency using fixed routing paths. As a result, the forwarding nodes of fixed routing paths may drain out their battery power quickly. 
Rana et al. presented an $\mathrm{A}^{*}$ algorithm based Energy Efficient Routing (ASEER) protocol to find optimal route in order to extend network lifetime [12]. In addition to estimated cost ' $h(n)$ ' and cost-so-far ' $g(n)$ ', the heuristic function in ASEER introduces a new metric ' $l(n)$ ' that denotes the path cost count of weak nodes having less energy. The ' $l(n)$ ' parameter is used to keep track of total number of low energy nodes in a current path. However, the protocol may not perform well if there exists multiple paths with same number of low energy nodes. As an example, if the $l(n)$ value of two available paths is equal, then the path with least $f(n)$ value will be selected. But, it may happen that the energy level of nodes in selected path is lower than the alternative path since ASEER protocol can't distinguish between the energy level of two nodes if both of them are below threshold level.

A new routing protocol based on fuzzy approach and $\mathrm{A}^{*}$ search algorithm is proposed in [13]. The routing scheme is capable of selecting optimal path between two nodes by favouring the lowest traffic load, minimum number of hops and highest level of residual energy. However, the protocol assumes that all the nodes are stable in terms of energy until the nodes are out of power. But, in real applications, there may be one or more intermittent sensor nodes that can add performance noise in the sensor networks. Furthermore, the performance of the protocol is not compared with any existing protocol.

To extend the network lifetime and to avoid network partitioning, we propose a new routing protocol using energy-based heuristic function and $\mathrm{A}^{*}$ search algorithm. The proposed routing algorithm uses residual energy of SNs as one of the route selection parameters when the energy level of a node is below the threshold level. In this way, EAHR protocol selects alternative routes and avoids the low energy nodes from being repeatedly used for data transmission.

\section{ENERGy AWARE HEURISTIC-BASED Routing PROTOCOL}

The network model used in this paper and some considerations of developing an efficient routing protocol are described. Then, the proposed EAHR protocol is presented in details.

\section{A. Network Model}

We assume that WSN comprises of several hundred nodes. We further assume that WSN has following properties.

- $\quad$ Every $\mathrm{SN}$ is assigned a unique node ID.

- Every SN knows the location of BS as well as its own.

- $\quad$ SNs periodically send the energy information to all of their neighbour nodes.

- SNs are equipped with limited and usually nonrechargeable batteries.

- The network consists of multiple stationary/mobile nodes and the energy consumption is not uniform for all nodes.

- $\quad$ SNs have different levels of transmission power and each node can dynamically adjust the power level [14].
Furthermore, we use the following model for radio hardware energy dissipation [15]. The transmitter consumes energy to run radio electronics and power amplifier. On the other hand, the receiver expends energy to run the radio electronics. We used free space ( $d^{2}$ power loss) and multipath fading $\left(d^{4}\right.$ power loss) channel models based on the distance between transmitter and receiver. Power control can be used to invert this loss by appropriately setting the power amplifier if the distance is less than a threshold $\left(d_{0}\right)$, the free space $(f s)$ model is used; otherwise, the multipath ( $\mathrm{mp}$ ) model is used. Therefore, in order to transmit $k$-bit message over the distance $d$, the radio hardware dissipates energy as per following equation (1).

$$
E_{T x}(k, d)=\left\{\begin{array}{cc}
k \times E_{\text {elec }}+k \times \epsilon_{f s} d^{2}, & d<d_{0} \\
k \times E_{\text {elec }}+k \times \epsilon_{m p} d^{4}, & d \geq d_{0}
\end{array}\right.
$$

and to receive this message, the radio expends:

$$
E_{R x}(k)=k \times E_{\text {elec }}
$$

The electronics energy, $E_{\text {elec }}$, is influenced by several factors such as: digital coding, filtering, modulation, and spreading of signal. On the other hand, the amplifier energy $\epsilon_{f s} d^{2}$ or $\epsilon_{f s} d^{4}$ is affected by factors such as acceptable bit-error rate and the distance between sender and receiver.

\section{B. The EAHR Protocol}

In this section, we describe the proposed routing protocol.

1) Heuristic Function: The heuristic function provides a good estimate of how far the destination node from the current node and thus helps to guide the search procedure. A number of parameters can be used in heuristic function that will collectively represent a numerical value. Since heuristic search is dependent on the heuristic function, it should be fine tuned to proceed the searching process in right direction. The EAHR protocol uses three different parameters to find the forwarding path. Longevity factor, distance from source node to current node and euclidean distance from current node to the BS are used to compute the heuristic value as follows:

$$
f(n)=h(n)+g(n)+100 / L F
$$

Here, ' $f(n)$ ' denotes heuristic value of node $n$, ' $h(n)$ ' is the estimated cost from node $n$ to the BS and ' $g(n)$ ' is the cost from node $n$ to current node. The longevity factor ' $L F$ ' is calculated according to the following equation:

$$
L F=\left(T \times E_{\text {res }}\right) / E_{\text {init }}
$$

where, $T$ is the sustainable time, $E_{\text {res }}$ denotes the residual energy and $E_{\text {init }}$ indicates the initial energy of SNs. The longevity factor is used to maximize the value of heuristic function when the energy of a forwarding node is below the threshold level.

2) $A^{*}$ Heuristic Search Algorithm: A* uses best-firstsearch technique to find a least-cost path from source to destination node. It first searches the path that appears to be most likely to lead towards the goal on the basis of heuristic function. The available node with the smallest value of ' $f$ ' is the node that should be expanded next. To find an optimal path to the destination using $\mathrm{A}^{*}$ search, ' $h(n)$ ' should be any lower bound of the actual distance to the destination node [16]. Thus, for an application such as routing, ' $h(n)$ ' 
might represent the Euclidean distance to the goal which is the shortest real distance between any two nodes. Here, we present the pseudocode of $\mathrm{A}^{*}$ search algorithm.

\section{Algorithm: A* heuristic search algorithm}

INPUT: start_node, goal_node

OUTPUT: path_list

\section{STEPS:}

closed_list $=\{$ the_empty_set $\} \quad$; list of visited node open_list $=\{$ start_node $\}$; list of tentative nodes to be visited $T \_v a l=$ set_threshold_level path_list $=$ the_empty_list ; empty list of node g_val[start_node] $=0 ;$; cost from start to best known path

if eng[start_node] $<T_{\text {_val }}$

$f \_$val[start_node] $=$g_val[start_node] $+h \_v a l\left[s t a r t \_n o d e\right]$

else

$$
+100 /\left(\left(\mathrm{T} \times \mathrm{E} \_ \text {res }\right) / \bar{E}_{-} \text {init }\right)
$$

$f \_$val $[$start_node $]=g \_v a l\left[s t a r t \_n o d e\right]+h \_v a l\left[s t a r t \_n o d e\right]$ end_if_else

while open_list is not empty

current_node $=$ the node in open_list with lowest $f \_v a l[]$

if current_node $==$ goal_node

return create_path_list(path_list, goal_node)

end_if

remove current_node from open_list

add current_node to closed_list

for each neighbor in neighbor_nodes[current_node]

tentative_g_val $=$ g_val[current_node] + dist_between(current_node,neighbor)

if neighbor in closed_list and tentative_g_val $\geq$ g_val[neighbor]

continue

end if

if neighbor not in closed_list OR

tentative_g_val $<$ g_val[neighbor] path_list[neighbor] = current_node g_val[neighbor] = tentative_g_val

if eng[neighbor] $<T$ _val

f_val[neighbor] = g_val[neighbor] +

$\bar{h} \_$val[neighbor] $+100 /\left(\left(\mathrm{T} \times \mathrm{E} \_\right.\right.$res $) / E \_$init $)$

else

f_val[neighbor] = g_val[neighbor] +

end_if_else h_val[neighbor]

end_if

if neighbor not in open_list end_if add neighbor to open_list

end for

end_while

return Not Found procedure create_path_list(path_list, current_node)

if current_node in path_list

$p=$ create_path_list(path_list, path_list[current_node]) return $(p+$ current_node $)$

else

return current_node

end_if_else

3) Routing Procedure: In our proposed EAHR protocol, the heuristic function takes the distance already travelled $[g(n)]$, the estimated distance to BS $[h(n)]$ and the longevity factor ' $L F$ ' to measure the heuristic value $[f(n)]$. In addition to local and global information, energy level (in the form of longevity factor) of each node is also considered to find the route from source to destination node. Furthermore, we use a threshold level to ensure optimal routing when the forwarding nodes have sufficient level of energy to route the data packets. If the energy level goes below the threshold level the algorithm tries to find alternative paths in order to extend the lifetime of the low power node.

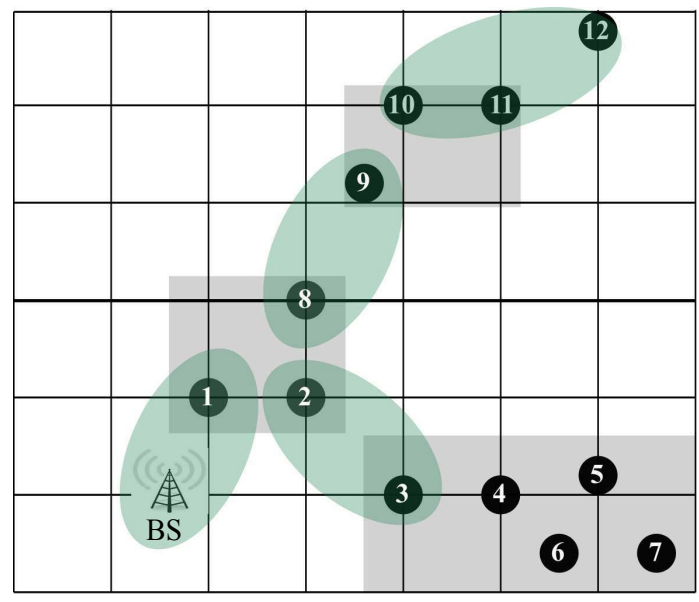

Fig. 1: Example of EAHR topology

Let us consider the above network topology with twelve sensor nodes and one sink node. The sensor nodes periodically send data packets to the BS. Each node is assigned a heuristic value ' $f$ ' used to guide the search process. In the figure, the area confined by rectangular and oval shapes represents the full connectivity among all nodes inside that region. Before sending data packets to the $\mathrm{BS}$, a node computes the route according to the algorithm described in previous section. Suppose, node 12 has to find a route to the BS. Initially, from all its neighbours ( 10 and 11 ), node 12 chooses one with the smallest heuristic values as its next hop. At this point, the energy level of each neighbour node is compared to the threshold level. If the energy of any node is below the threshold level, the heuristic value of that node is incremented according to energy-based heuristic function. Since, $A^{*}$ search algorithm selects the node with lowest heuristic value as next hop, the low power node can easily be avoided if there exists an alternative path with least heuristic value. For example, consider the following initial parameters: $\{[g(10)=3, g(11)$ 
$=1.5] ;[h(10)=10, h(11)=10.5] ;[\operatorname{eng}(10)=0.5$, eng $(11)=$ $0.5], T=50$ minutes $\}$ and the threshold level is set to 0.25 . Hence, we get the $f$-value for these two nodes as follows: $\{f(10)=13$ and $f(11)=12\}$. Since the $f$-value of node 11 is smaller than that of node 10 , node 12 selects node 11 as forwarding node. However, during next hop selection process, the algorithm computes the $f$-value for node 9 via both node 10 and 11. Let us say the estimated cost of node 9 to the BS is 8 and the direct distance to node 10 and node 11 is 1 and 3 respectively. Hence, the $f$-value to reach node 9 is 12 through node 10 and 12.5 through node 11 respectively. Since, the route via node 10 is smaller than the previous route, node 12 updates its routing table after receiving the new route information. In this way, node 12 finds the optimal route to the BS using $A^{*}$ search algorithm and uses the route to send data packets until the energy level of node 10 goes below the threshold level.

After time $t$, we assume that the energy level of node 10 and 11 degrades to 0.2 and 0.4 joules respectively. Since, the energy level of node 10 is below the threshold level, node 12 recomputes the heuristic value of node 10 using the heuristic function. The new $f$-value of node 10 is: $f(10)=3+10+$ $100 /((50 \times 0.2) / 0.5)=18$. Since the value is greater than that of node $11[f(11)=12]$; node 11 is selected as the forwarding node by node 12. Thus, the proposed protocol follows optimal route when the nodes have sufficient level of energy. Otherwise, it searches for alternative paths to avoid low energy node and thus extends the network lifetime.

\section{EXPERIMENTS AND ANALYSIS}

We have simulated the proposed EAHR algorithm and compared the results with WSNHA-GAHR (Greedy and A* Heuristic Routing for Wireless Sensor Network in Home Automation) protocol since both EAHR and GAHR protocols are suitable for home automation. For our experiments, we have deployed two different scenarios with 25 and 13 nodes in a $50 \times 50\left(\mathrm{~m}^{2}\right)$ simulation area. The transmission range of all sensor nodes is fixed to $15 \mathrm{~m}$. Here, we have detailed the scenarios.

Scenario 1: In this scenario, we have analysed the total energy dissipation, network lifetime, impacts of dead nodes, unused energy and message throughput. The simulation parameters used in the experiments are listed in the following table.

TABLE I: Simulation parameters used in the experiments

\begin{tabular}{|c|c|c|}
\hline Types & Parameters & Value \\
\hline \multirow[t]{3}{*}{ Network } & Network Grid & $(0,0)$ to $(50,50)$ \\
\hline & Initial Energy & $0.35 \mathrm{~J}$ \\
\hline & Location of BS & $(25,25)$ \\
\hline \multirow[t]{4}{*}{ Application } & Transmission Range & $15 \mathrm{~m}$ \\
\hline & Data Packet Size & 128 bytes \\
\hline & Broadcast Packet Size & 24 bytes \\
\hline & Packet Header Size & 24 bytes \\
\hline \multirow[t]{5}{*}{ Radio Model } & $E_{\text {elec }}$ & $50 \mathrm{~nJ} / \mathrm{bit}$ \\
\hline & $E_{f s}$ & $10 \mathrm{pJ} / \mathrm{bit} / \mathrm{m}^{2}$ \\
\hline & $E_{m p}$ & $0.0013 \mathrm{pJ} / \mathrm{bit} / \mathrm{m}^{4}$ \\
\hline & EDA & $5 \mathrm{~nJ} / \mathrm{bit} /$ signal \\
\hline & Threshold Distance $\left(d_{0}\right)$ & $87.71 \mathrm{~m}$ \\
\hline
\end{tabular}

The effects of the dead nodes on the whole network is presented in Fig. 2. The figure indicates that both protocols have same impact on the rest of the nodes in the network till the loss of power of eight nodes. After that, the number of affected nodes in GAHR is higher than EAHR protocol. The same result is observed until the loss of power of twelve nodes in total. In EAHR protocol, low energy nodes are avoided if there exists alternative path to route the data packets. As a result, nodes remain alive for more rounds and minimizes the number of affected nodes. For example, only two nodes are affected in EAHR protocol when nine nodes out of twenty five completely run out of their battery power, whereas, the number of affected nodes is nine in GAHR protocol.

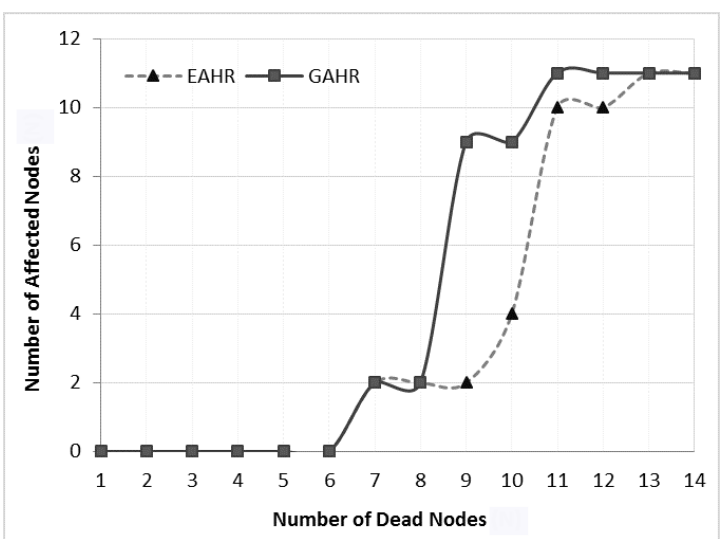

Fig. 2: Impacts of dead nodes in the network

Fig. 3 illustrates the simulation result on the network lifetime comparison of EAHR and GAHR protocols. From the graph, we see that the first node depletes its power after 188 rounds in GAHR while all nodes in EAHR protocol are alive till 396 rounds. Finally, before the network is being disfunctional (i.e., fourteen nodes are out of power), nodes in EAHR protocol sustain more than 200 rounds compared to GAHR algorithm.

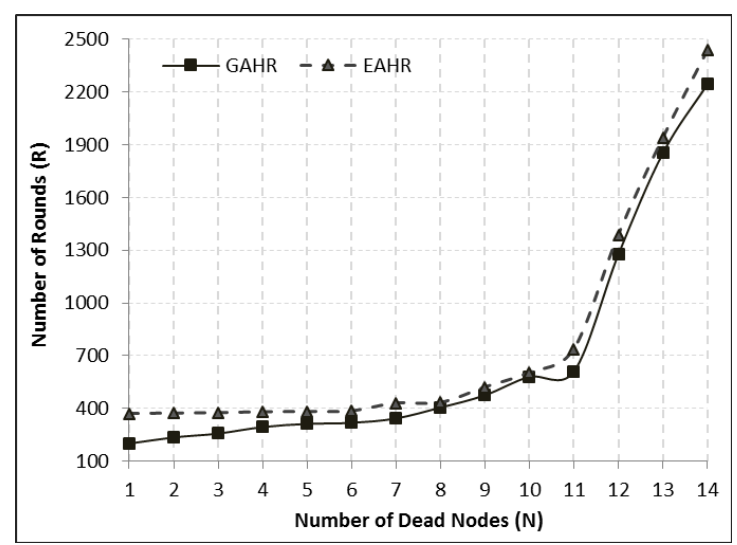

Fig. 3: Network lifetime (Scenario 1)

The Fig. 4 shows the total energy dissipation for both protocols. EAHR dissipates more energy than GAHR since nodes are alive for more rounds and send information to 
the BS. Most of the WSN applications have to send data packets to the BS through multi-path routing. If any node in the routing path exhausts its power quickly, it may partition the network. The other nodes that forward information via exhausted node may unable to communicate with the BS, although the nodes still have significant amount of energy. This scenario is very common in GAHR protocol.

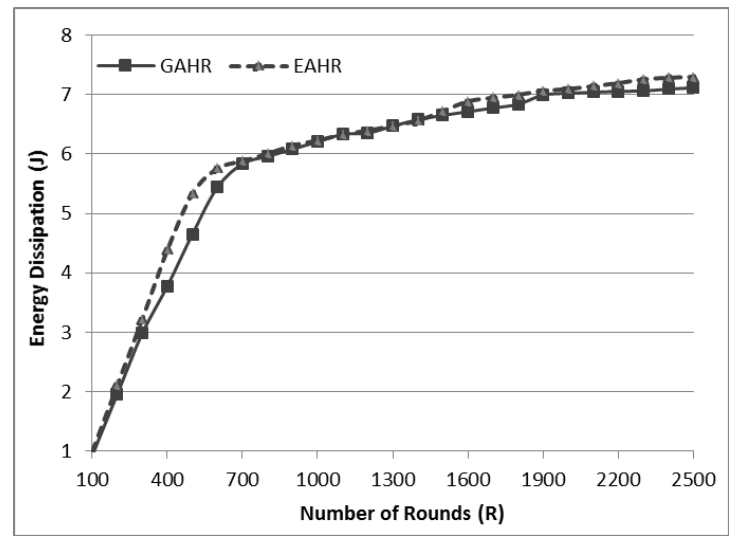

Fig. 4: Total energy dissipation

In Fig. 5, the amount of unused energy in GAHR and EAHR protocols is represented. From the graph, we see that more energy is unused in GAHR compared to EAHR routing scheme. This is because the node failures in GAHR may divide the entire network, the remaining energy of the disjointed nodes is completely unused if the network path can not be repaired.

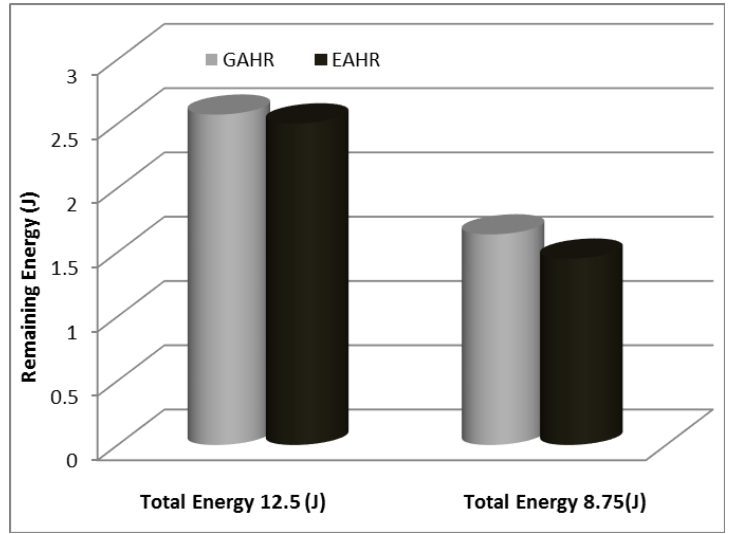

Fig. 5: Unused energy

Finally, we have examined the packet delivery ratio of our proposed EAHR protocol by sending different number of data packets to the BS as shown in Fig. 6. In the first phase, the sensor nodes sent a total of 1000 data packets. In GAHR, the BS successfully received 924 of these packets, resulting a packet delivery ratio of $92.4 \%$. For the same number of data packets, the BS in EAHR received 966, resulting a packet delivery ratio of $96.6 \%$. Similarly, in phase 2 , the sensor nodes sent a total of 2000 data packets. Out of these packets, 1746 packets are received by the BS in GAHR, for a packet delivery ratio of $87.3 \%$, whereas, the packet delivery ratio of EAHR is $93.85 \%$ for successfully receiving 1877 data packets. Finally, a total of 3000 data packets sent to the BS. The BS received 2609 in GAHR and 2791 data packets in EAHR correctly, resulting a packet delivery ratio of $86.96 \%$ and $93.03 \%$ respectively.

The simulation results show that the packet delivery ratio in EAHR is higher than that of GAHR. The reason is that some of the forwarding nodes in GAHR protocol quickly loose power. As a result, the packets transmitted by the nodes of disjointed area are not received by the BS.

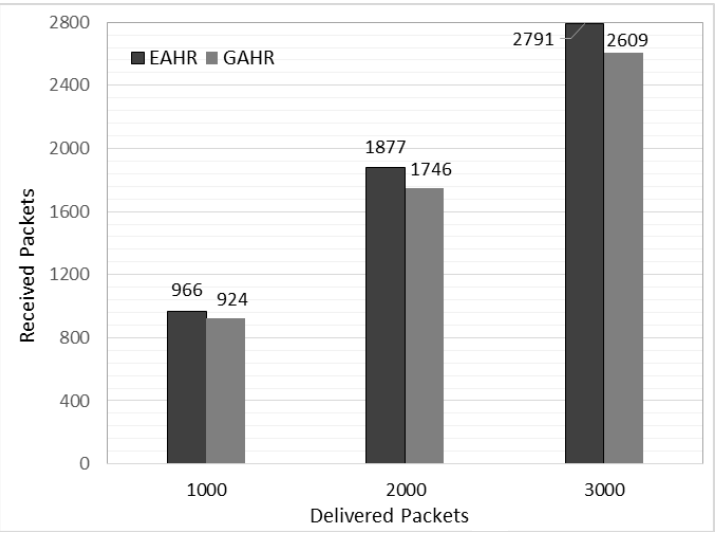

Fig. 6: Throughput comparison

Scenario 2: We have also simulated the following network in OMNET++ to evaluate the sustainability of the proposed protocol where network may suffer from bottleneck performance. For our experiments, the energy level of every node is set to 2 joules initially.

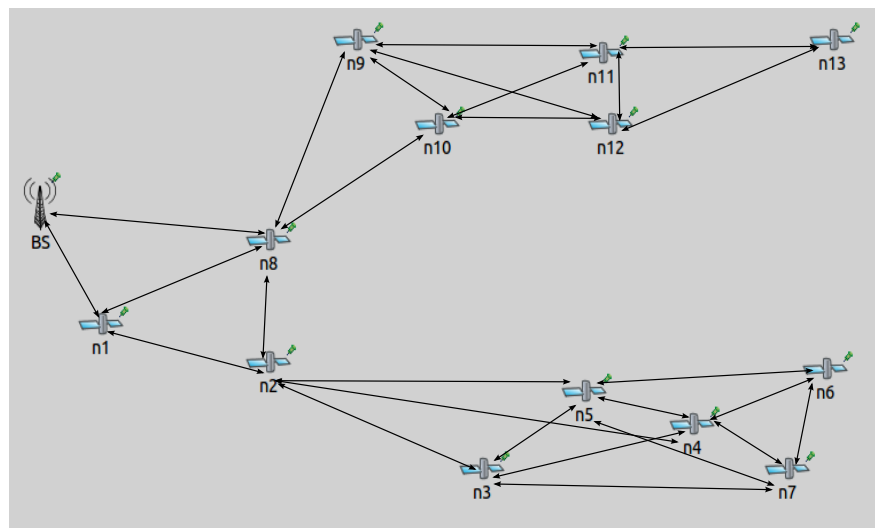

Fig. 7: A sensor network with 13 nodes

The following graphs represent the simulation outcomes obtained from the experiments. Fig. 8 presents the number of rounds until the network becomes disfunctional. The first node (node 8 ) exhausts its power after 429 rounds in GAHR while second (node 2) and third node (node 1) are out of power after 902 and 4174 rounds respectively. On the other hand, EAHR executes 862 rounds before the power outage of first node. Second and third node deplete their battery life after 917 and 2962 rounds respectively. This is because EAHR 
follows optimal route until the energy level of a node goes below the threshold level. When the power of a node is below the threshold level, it finds alternative path and thus avoids the low power node from being selected as a forwarding node. But, GAHR uses greedy forwarding and selects the same route to forward data packets without noticing the energy level of the forwarding nodes. As a result, the forwarding nodes quickly exhaust their power and partition the network.

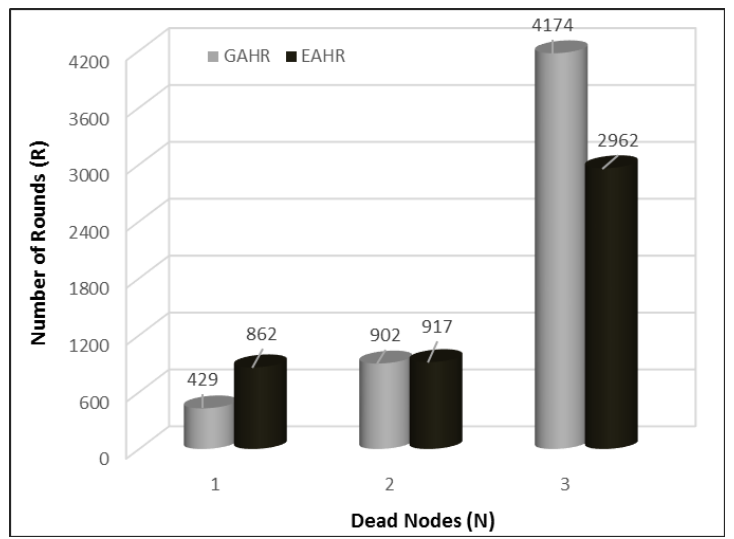

Fig. 8: Network lifetime (Scenario 2)

In the graph, we see that after 429 rounds in GAHR, node 8 exhausts its energy and as a consequence node 9-13 can not communicate with the BS. Similarly, node 3-7 are disconnected after 902 rounds when the power of second node i.e., node 2 is drained. At this point, all the nodes except node 1 are disjointed from the BS. This single node remains alive till 4174 rounds in GAHR.

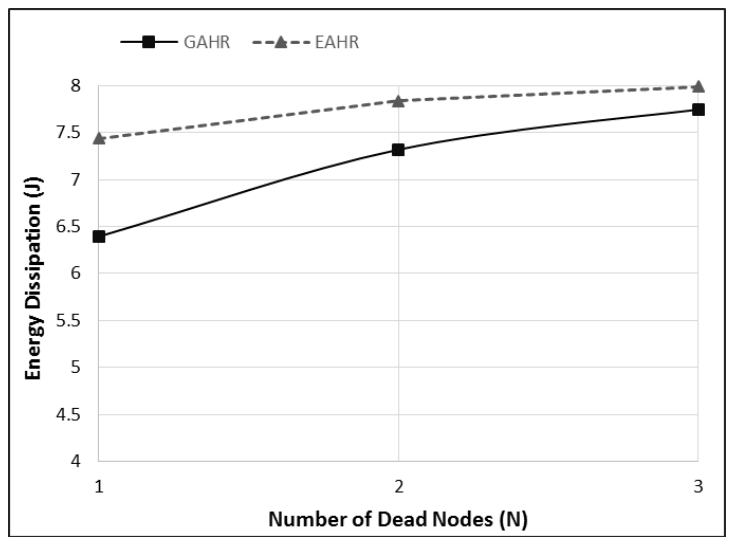

Fig. 9: Total energy dissipation (Scenario 2)

From the results shown in Fig. 9, it is observed that significant amount of energy is dissipated in EAHR protocol. GAHR consumes about 6.4 joules energy before the first node dies whereas the amount is about 7.4 joules for EAHR. The amount of unused energy is 18.25 joules in GAHR, whereas, it is 18.01 joules in EAHR. Thus, EAHR protocol utilizes the energy of the sensor nodes more efficiently than GAHR protocol and increases the overall network lifetime.

\section{Conclusion}

In this paper, an energy aware heuristic-based routing protocol is proposed that uses heuristic function and $\mathrm{A}^{*}$ search to find an optimal route. In addition to local and global information, longevity factor is used as one of the route selection parameter in our proposed algorithm. Hence, EAHR protocol always avoids the low-energy nodes if there exists alternative paths in the network. Our proposed routing scheme extends network lifetime and degrades the chance of network partition. Simulation results show that the network sustains for more rounds and most of the nodes are functional in EAHR compared to GAHR protocol. The proposed protocol is suitable for a small network (e.g., home automation) and can also be implemented in large scale sensor networks (e.g., environmental monitoring). Our future work is to integrate new routing parameters in heuristic function in EAHR protocol to observe the impacts of bandwidth, number of hops and link quality.

\section{REFERENCES}

[1] G. R. Sakthidharan and S. Chitra, "A survey on wireless sensor network: An application perspective," in ICCCI, pp. 1-5, 2012.

[2] K. Biswas, V. Muthukkumarasamy and E. Sithirasenan, "Maximal clique based clustering in WSNs," in ISSNIP, pp. 237-241, 2013.

[3] H. Zhang and H. Shen, "Balancing energy consumption to maximize network lifetime in data-gathering sensor networks", in IEEE Trans. Parallel Distrib. Syst., vol. 20, pp. 1526-1539, 2009.

[4] K. Akkaya and M. Younis, "A survey of routing protocols in wireless sensor networks", in Ad Hoc Network, vol. 3(3), pp. 325-349, 2005.

[5] F. Ren, J. Zhang , T. He , C. Lin and S. K. Das, 'EBRP: Energy-balanced routing protocol for data gathering in wireless sensor networks", in IEEE Trans. Parallel Distrib. Syst., vol. 22, pp. 2108 -2125, 2011.

[6] K. Biswas, V. Muthukkumarasamy and E. Sithirasenan, " An energy efficient clique based clustering and routing mechanism in WSNs", in IWCMC, pp. 171 - 176, 2013.

[7] B. Karp and H. T. Kung, "GPSR: Greedy perimeter stateless routing for wireless networks," in MobiCom, 2000.

[8] D. Estrin Y. Yu and R. Govindan, "Geographical and energy-aware routing: A recursive data dissemination protocol for wireless sensor networks", in Tech. Report, 2001.

[9] X. H. Li, S. H. Hong, and K. L. Fang, "WSNHA-GAHR: A greedy and $A^{*}$ heuristic routing algorithm for wireless sensor networks in home automation," in IET Comm., vol. 5(13), pp. 1797-1805, 2011.

[10] J. Park and S. Sahni, "An online heuristic for maximum lifetime routing in WSNs", in IEEE Trans. Comput., vol. 55, pp. 1048-1056, 2006.

[11] C. Wu , R. Yuan and H. Zhou, "A novel load balanced and lifetime maximization routing protocol in WSNs," in Proc. IEEE Vehicular Tech. Conf. pp. $113-117,2008$.

[12] K. M. Rana and M. A. Zaveri, "ASEER: A routing method to extend life of two-tiered wireless sensor network", in Int. J. Adv. Smart Sensor Netw. Syst., vol. 11(2), pp. 1 -16, 2011.

[13] I. S. AlShawi, Y. Lianshan, W. Pan and B. Luo, "Lifetime enhancement in wireless sensor networks using fuzzy approach and A-Star algorithm", in IEEE Sensors J., vol. 12(10), pp. 3010-3018, Oct. 2012.

[14] O. Younis and S. Fahmy, "HEED: A hybrid, energy-efficient, distributed clustering approach for ad hoc sensor networks", in IEEE Trans. on Mobile Computing, vol. 3(4), pp. 366-379, 2004.

[15] W. B. Heinzelman, A. P. Chandraprakasan and H. Balakrishnan, "Application specific protocol architecture for wireless microsensor networks", in IEEE Trans. on Wireless Networking, vol. 1(4), pp. 660-670, 2002.

[16] K. M. Passino and P. J. Antsaklis, "A metric space approach to the specification of the heuristic function for the algorithm", in IEEE Trans. on System, MAN and Cybernetics, vol. 24(1), pp. 159-166, 1994. 